Purpose To demonstrate the cost of care by embolization of intracranial aneurysm and to understand relation between the cost and clinical patient parameters.

Materials and Methods Between January 2010 and April 201248 patients were treated by embolization of cerebral aneurysms. The cost of pharmaceutical products (drugs and medical devices) was assessed by using the micro-costing method that takes into account all direct costs and the overall cost of care was calculated using data from the hospital's information system.

Results In total, 48 patients were treated, mean age $52.4 \pm 12.5$ years. The sex ratio $\mathrm{M} / \mathrm{F}=0.71 .26$ patients were covered by health insurance $(52.2 \%)$. The median overall stay within 10 days [5-11] in ICU was 1 day [1 to 2] and in the medical unit was 6 days [3 to 9.75]. The overall average cost of treatment was $€ 9,697.8$, varying from $€ 4,784.3$ to $€ 32,172.3$. The cost of pharmaceutical products was on average $57.6 \%$ of the overall cost. While the average cost of consumables was $€ 5,612.4$ with a range of $€ 2,499.1$ to $€ 16,370$. . Length of stay does not influence the overall cost of care, but the cost is influenced by the amount of embolization material.

Conclusions The cost of pharmaceutical products in the endovascular treatment of intracranial aneurysms remains high and represents a major handicap for the development of this technique in countries with low coverage by health insurance. As we mentioned before, this latter overall cost is especially influenced by number of embolization materials and number of aneurysms.

No conflict of interest.

\section{OHP-076 THE INCIDENCE OF BACTEREMIA DUE TO CATHETERS AND THE COST OF ANTIBIOTICS BEFORE AND AFTER IMPLEMENTATION OF THE ZERO BACTEREMIA PROJECT}

doi:10.1136/ejhpharm-2013-000276.449

'A Morego Soler, 'A Bosó-Ribelles, 'I Sanchez-Martinez, 'B Arribas-Diaz, 'JC TitosArcos, 'MM Sanchez-Catalicio, 'MP Molina-Guillen, ${ }^{2} \mathrm{~B}$ Gil-Rueda, 'MC SanchezMulero. 'N Manresa-Ramón. 'Hospital Morales Meseguer, Hospital Pharmacy, Murcia, Spain: ${ }^{2}$ Hospital Morales Meseguer, Intensive Care Unit, Murcia, Spain

Background Primary bacteraemia and bacteraemia caused by catheter infections entail a high pharmaceutical cost. The 'Zero Bacteraemia Project' (BZP) for central intravenous catheter (CVC) use in invasive therapies showed a decrease in the number of bacteraemia cases and a financial effect on hospitalizations.

Purpose To study the number of primary bacteraemia and bacteraemia cases caused by catheter infections among patients hospitalised in our Intensive Care Unit (ICU) and the pharmaceutical cost after implementation of the CVC guides. We compared these data to those obtained from 2007-2008.

Materials and Methods We retrospectively studied 2353 patients who were admitted to our Intensive Care Unit. 1280 patients were studied before BZP (2007-2008) and 1073 after BZP implementation. The BZP implied: catheter insertion with maximal sterile barrier precautions in ICU, correct hand washing, hygienic precautions when using CVCs and the removal of unnecessary catheters. We compared the pharmaceutical cost in antibiotics in both periods. We also studied the five most-used antibiotics in this hospital for the treatment of catheter-related infections suffered by the sample group in this ICU. The data were obtained by the programme 'ENVIN-ICU'.

Results A total of 35 pre-BZP and 13 post-BZP catheter-related bacteraemia cases were detected. 5.14 and 2.17 bacteraemia cases for every 100 patients with CVC. A 37\% reduction was observed in the incidence of bacteraemia. The pharmaceutical cost just in antibiotics for the 35 patients infected during the first period amounted to 3100.68 euros. However it dropped to 2388.93 euros during the following period. A $23 \%$ saving was observed on the antibiotics consumption.
Conclusions The data from this study show that the use of the 'Zero Bacteremia' policy in the process of inserting and monitoring CVCs is useful to reduce the number of infections. A statistically significant decrease in the number of bacteraemia cases and a monetary saving in antibiotics were found too.

No conflict of interest.

\section{OHP-077 THE ROLE OF PHARMACISTS IN AN ITALIAN MODEL OF ECONOMIC SUSTAINABILITY AND INNOVATIVE TREATMENTS}

doi:10.1136/ejhpharm-2013-000276.450

${ }^{1} \mathrm{~S}$ di Pietro, ${ }^{2 M}$ Salanitro, ${ }^{3}$ R Rapisarda. 'Azienda Sanitaria Provinciale Siracusa, Hospital Pharmacy Lentini/Pharmacy Department, Lentini, Italy; ${ }^{2}$ Azienda Sanitaria Provinciale Catania, Hospital Pharmacy Acireale/Pharmacy Department, Catania, Italy; ${ }^{3}$ Azienda Sanitaria Provinciale Catania, Pharmacy Department, Catania, Italy

Background Italy is one of the European countries where a Risk Sharing Scheme between healthcare institutions and pharmaceutical companies has been widely implemented. It is a new model proposed to accelerate the authorisation and the availability on the market of new drugs. Since September 2007, the Italian drug agency has developed a web register to record data to monitor patients who receive medicines under a Risk Sharing Scheme: the physician prescribes medicines from a list of high-cost oncology drugs and the Italian drug agency validates each prescription and e-mails the hospital pharmacy to release the drug. The non-responding patients are documented in the web register by the health authorities and the pharmacist applies for reimbursement to the pharmaceutical company. Since 2011, Sicily Region has had a hospital pharmacist officially appointed in each pharmacy department to be in charge of obtaining refunds from manufacturers for undocumented nonresponding patients and to supervise the pay-back procedures.

Purpose To quantify the amount clawed back from manufacturers after the appointment of the Risk-Sharing pharmacist.

Materials and Methods We detected and examined unresponsive patients recorded in the Registro AIFA-onco. The pay-back procedures were subsequently completed.

Results The number of registered patients increased by $83 \%$ and 451 non-documented patients were recorded: 190 Erlotinib, 103 Sorafenib, 57 Sunitinib, 38 Lapatinib, 14 Everolimus, 1 Pemetrexed, 20 Bevacizumab, 20 Cetuximab, 12 Gefitinib, 2 Vinflunine, 16 Lenalidomide, 3 thalidomide, 1 Panitumumab, 7 Bortezomib, 4 Azacitidine, 3 Trabectedin.

The ex-factory expense was $€ 6,340,011.66$ : $€ 431,063.89$ recovered, $€ 145,678.92$ is waiting for reimbursement and $€ 136,220.50$ has been denied reimbursement.

Conclusions The appointment of a pharmacist enabled us to monitor pay-back procedures and assess responding and nonresponding patients reliably.

No conflict of interest.

\section{OHP-078 TREATMENT OF POSTOPERATIVE ANAEMIA IN ORTHOPAEDIC SURGERY: A BUDGET IMPACT ANALYSIS FROM A HOSPITAL PERSPECTIVE}

doi:10.1136/ejhpharm-2013-000276.451

JV Chauny, A Razurel, B Politis, F Le Mercier. AP-HP Hôpital Ambroise-Paré, Pharmacie, Boulogne-Billancourt, France

Background Standard postoperative anaemia management includes oral iron or intravenous iron supplementation (iron sucrose complex, ISC), erythropoietin therapy and blood transfusion. Introduction of a new intravenous iron formulation (ferric carboxymaltose, FCM), more expensive than ISC but with simplified administration modalities, could have economic consequences for hospitals. 